\title{
La precariedad laboral por grupos ocupacionales en el Estado de México, 2005 y 2015
}

\author{
Job insecurity by occupational groups \\ in the State of Mexico, 2005 and 2015
}

\author{
Sergio Cuauhtémoc Gaxiola Robles Linares* (iD http://orcid.org/0000-0002-7782-9058 \\ Daniel Lozano Keymolen** (D) http://orcid.org/0000-0003-1086-7233 \\ Bernardino Jaciel Montoya Arce *** $^{* *}$ https://orcid.org/0000-0002-2794-0997 \\ Yuliana Gabriela Román Sánchez *** (iD http://orcid.org/0000-0001-8571-9660
}

\begin{abstract}
Resumen
En este artículo se analiza la precariedad laboral por grupos ocupacionales de trabajadores asalariados en el Estado de México, en 2005 y 2015. Se utilizó la técnica estadística del análisis por conglomerados, para agrupar a dichos trabajadores en cuatro grupos de precariedad: alto, medio, bajo y no precario, con base en la Encuesta Nacional de Ocupación y Empleo del segundo trimestre de 2005 y 2015. La población objetivo se restringe a los trabajadores asalariados, y el análisis de la precariedad laboral se realiza con variables generales, sin profundizar en casos específicos, a través de la metodología cualitativa. La originalidad aquí es que el análisis de la precariedad se hizo en un contexto regional, y también que es un estudio comparativo entre 2005 y 2015 . Uno de los resultados más relevantes es la concentración de los trabajadores asalariados en los grupos extremos: precario alto y no precario, lo que muestra la polarización laboral en el mercado de trabajo del estado.

Palabras clave: precariedad laboral; empleo precario; desigualdad laboral; análisis de conglomerados; relaciones laborales; Estado de México.
\end{abstract}

\begin{abstract}
This article analyzes job insecurity by occupational groups of salaried workers in the State of Mexico, in 2005 and 2015. The statistical technique of cluster analysis was used, grouping these workers into four precariousness groups: high, medium, low and not precarious, based on the National Survey on Occupation and Employment of the second semester of 2005 and 2015. Target population is limited to salaried workers, and job insecurity is analyzed using standard variables, without delving into specific cases, through qualitative methodology. The originality of this study is that job insecurity was analyzed in a regional context; moreover, this is a comparative study between 2005 and 2015. One of the most relevant results is the concentration of salaried workers in the extreme groups: high precarious and not precarious. This shows labor polarization existing in the state's labor market.

Keywords: job insecurity; precarious employment; labor inequality; cluster analysis; labor relations; State of Mexico.
\end{abstract}

Cómo citar: Gaxiola Robles Linares, S. C., Lozano Keymolen, D., Montoya Arce, B. J.,y Román Sánchez,Y. G. (2019). La precariedad laboral por grupos ocupacionales en el Estado de México, 2005 y 2015. región y sociedad, 3 I, e986. doi: I0.22 I 98/rys20 I 9/3 I/986

* Autor para correspondencia. Universidad Autónoma del Estado de México, Centro de Investigación y Estudios Avanzados de la Población. Paseo Tollocan s/n. Cerro de Coatepec, Ciudad Universitaria, C. P. 50110, Toluca, Estado de México, México. Teléfono: (722) 215 7111. Correo electrónico: serobles99@gmail.com

** Universidad Autónoma del Estado de México, Centro de Investigación y Estudios Avanzados de la Población. Paseo Tollocan s/n. Cerro de Coatepec, Ciudad Universitaria, C. P. 50110, Toluca, Estado de México, México. Teléfono: (722) 2157111. Correo electrónico: daniel. lozkey@gmail.com

*** Universidad Autónoma del Estado de México, Centro de Investigación y Estudios Avanzados de la Población. Paseo Tollocan s/n. Cerro de Coatepec, Ciudad Universitaria, C. P. 50110, Toluca, Estado de México, México. Teléfono: (722) 2157111. Correo electrónico: bjmontoyaa@uaemex.mx

**** Universidad Autónoma del Estado de México, Centro de Investigación y Estudios Avanzados de la Población. Paseo Tollocan s/n. Cerro de Coatepec, Ciudad Universitaria, C. P. 50110, Toluca, Estado de México, México. Teléfono: (722) 2157111. Correo electrónico: madon.dl26@gmail.com

Recibido: 22 de septiembre de 2017

Aceptado: 12 de marzo de 2018

Liberado: 16 de enero de 2019 


\section{Introducción}

La revolución industrial del siglo XIX provocó cambios en las relaciones de trabajo; surgió la clase del trabajador asalariado, y se consolidó un nuevo modelo económico mundial. Sin embargo, el colapso de éste, junto con las crisis económicas recurrentes que comenzaron a finales de la década de 1970, provocó modificaciones en los marcos normativos y de regulación de las relaciones de trabajo, que llevaron a un nuevo modelo económico, ${ }^{1}$ que tuvo repercusiones en el mercado y en las dinámicas laborales (Cervantes, 2017).

En este contexto, a partir de los años ochenta, con la implementación del modelo neoliberal, se trasformaron las dinámicas en los mercados de trabajo a través de la flexibilización laboral, que se caracteriza por una desregulación permanente de los procesos laborales y se vincula con la precariedad laboral, que fue resultado del funcionamiento social y económico de dicho modelo.

En este sentido, el origen de la flexibilidad y la precariedad laboral fue la reestructuración productiva y económica de alcance mundial. De tal manera que las estrategias empresariales llevaron a un abaratamiento de la mano de obra para reducir los costos de producción, lograr mayor competitividad y tener más ganancias de capital, a costa de una estructura ocupacional más precaria.

Diversas investigaciones muestran el deterioro de las relaciones laborales en el marco del nuevo modelo económico; en Estados Unidos, Reino Unido y Dinamarca (Kalleberg, 2009); Italia (Farcomeni, Nardi y Fabrizi, 2011); Túnez (Stampini y Verdier, 2011); Costa Rica (Mora, 2011); México (Rojas y Salas, 2011) y Panamá (Castillo, 2001) hay evidencias de cambios negativos en los mercados de trabajo.

Rojas y Salas (2011) fueron de los primeros en exponer el concepto de precariedad laboral en México; ellos intentaron mostrar la precarización del empleo en el periodo 1995-2004, a través de la inestabilidad (contrato temporal o verbal) y la inseguridad (acceso a la seguridad social), así presentaron la fragilidad de las relaciones laborales. Por ejemplo, en 2004 aproximadamente 50\% de los trabajadores asalariados tenían un empleo inestable (contrato temporal o verbal).

Las relaciones laborales en el Estado de México se han estudiado poco, por lo que esta investigación permitirá ahondar en la precariedad laboral y su efecto diferencial en los grupos ocupacionales.

De esta manera, se puede plantear la hipótesis de que la precariedad laboral es un fenómeno presente en el mercado de trabajo del Estado de México, y repercute en las relaciones, las prestaciones y los derechos laborales. De hecho, la entidad mexiquense constituye el mercado de trabajo más grande de la república mexicana, debido a la gran cantidad de población económicamente activa $\left(\right.$ PEA) ${ }^{2}$ (Sollova y Barrios, 2010).

1 "Un modelo económico es una descripción simplificada de la realidad, concebido para ofrecer hipótesis sobre conductas económicas que pueden comprobarse" (Oularis, 2011, p. 46).

2 La PEA abarca a todas las personas que se encuentran en edad de trabajar, que contaban con una ocupación en el periodo de referencia, o que no la tenían, pero estaban buscando emplearse con acciones específicas (Instituto Nacional de Estadística y Geografía [INEGI], 2002). 
Así, la importancia de estudiar la precariedad laboral radica en que provoca la pérdida del empleo, como un elemento cohesionador de la sociedad; el fenómeno ha tenido auge en las discusiones del mercado de trabajo del Estado de México y del país en los últimos diez años, por lo que se ha colocado como un referente importante sobre las relaciones laborales, los modos de producción, ${ }^{3}$ la organización del trabajo y la sindicalización, entre otros temas. De hecho, la precariedad laboral en México ha sido una constante, pues si bien tiende a aumentar durante los periodos de crisis económicas, se mantiene como una de las características principales del mercado laboral (Rubio, 2017).

En este contexto, el objetivo aquí es analizar la precariedad laboral por grupos ocupacionales de los trabajadores asalariados en el Estado de México, en 2005 y 2015, ${ }^{4}$ para conocer el efecto del fenómeno en la configuración de ellos.

El Estado de México se eligió como caso de estudio porque cuenta con el mercado laboral más grande del país, ${ }^{5}$ lo que permite profundizar en las modalidades de precariedad laboral, además en la entidad coexisten trabajadores de alta y baja calificación, ya que tiene zonas de desarrollo económico alto (cercanía a Ciudad de México), y otras con rezagos sociales relevantes (sur de la entidad).

En cuanto a la metodología, primero se utilizó un análisis por conglomerados para agrupar a los trabajadores asalariados mexiquenses, según sus relaciones laborales, en cuatro grupos de precariedad: alto, medio, bajo y no precario. Después se conformaron cuatro grupos ocupacionales (manual alta calificación, manual baja calificación, no manual alta calificación, no manual baja calificación), para estudiar la distribución de trabajadores precarios en las ocupaciones del mercado laboral, en 2005 y 2015. La fuente de información utilizada fue el segundo trimestre de la Encuesta Nacional de Ocupación y Empleo (ENOE, 2005 y 2015) (Lucatero, 2016).

El artículo se divide en cinco secciones, la primera incluye la revisión conceptual de la precariedad laboral, la segunda el contexto sociodemográfico y el entorno laboral de los trabajadores mexiquenses en 2005 y 2015. En la tercera se detalla la metodología y la fuente de información, en la cuarta el estudio de las cuatro relaciones laborales en que se basa la precariedad para esta investigación. En la quinta se presenta el análisis de la precariedad laboral por grupo ocupacional de los trabajadores asalariados mexiquenses en los años de análisis $y$, por último, las conclusiones.

3 Son los que permiten la producción de bienes y servicios necesarios en una sociedad, para garantizar su subsistencia.

4 Este periodo se eligió porque durante estos 10 años diversos estudios han mostrado que la precarización del empleo se ha acentuado en el Estado de México y en el país, por lo que profundizar en el análisis de este fenómeno contribuye a conocer las particularidades que adquiere. Además, de 2005 a 2015, el número de trabajadores en la entidad creció aproximadamente en dos millones, por lo que es importante conocer el tipo de empleo que se generó durante este lapso.

5 En 2015, la PEA en el Estado de México era de alrededor de 7 millones de personas. 


\section{Precariedad laboral: una revisión conceptual}

El trabajo es un elemento central que ha permitido el desarrollo social, económico, político y cultural del ser humano, fundamental para su existencia y sobrevivencia. De acuerdo con Marx, el trabajo es una parte esencial del individuo, pues señala que "al producir los medios de vida, el hombre produce indirectamente su propia vida material” (Marx, 1991, p. 47). En la actualidad, estudiar el mercado de trabajo permite comprender una parte esencial de la dinámica del proceso social, que es resultado de una serie de cambios en la economía mundial.

A partir de la segunda mitad del siglo XX, el trabajo a escala internacional constituyó un elemento generador de cohesión social, debido a que los beneficios que se otorgaban a los trabajadores les permitían gozar de cierta movilidad social. Sin embargo, en los años ochenta se implementó el modelo económico neoliberal, que trajo consigo trasformaciones en los mercados laborales que incidieron en la estructura, las relaciones, las condiciones y las dinámicas laborales desarrolladas hasta ese momento, entre ellas sobresalen: a) la flexibilidad y precariedad laboral, b) la incorporación de grupos nuevos de trabajadores (mujeres y jóvenes), c) el desempleo y la exclusión social, d) la externalización del trabajo y e) la inestabilidad laboral.

Entre estos cambios destaca la flexibilización creciente, caracterizada principalmente por la desregulación de los procesos laborales, uno de cuyos propósitos era reducir los costos en las empresas. Para Carnoy, la flexibilidad del empleo se entiende como el proceso en el cual "los cometidos laborales y el tiempo de trabajo pueden adaptarse constantemente a productos, procesos y mercados cambiantes" (2001, p. 79).

Con base en lo anterior, en los mercados de trabajo se modificaron las dinámicas laborales, mediante una reorganización productiva y empresarial orientada a cambiar los procesos de trabajo, que han permitido crear ocupaciones sobre todo en el sector terciario. El resultado de todos estos cambios fue la pérdida del carácter cohesionador que se adjudicaba tradicionalmente al trabajo, y que facilitaba la integración entre las capas sociales. En este sentido, el trabajo ha perdido valor y el trabajador se ha vuelto desechable, inseguro, flexible, sin identidad ni autonomía.

Las modificaciones en los mercados de trabajo deterioraron las relaciones laborales y provocaron una precarización del empleo, como ya lo preveían Froebel, Heinrichs y Kreye (1981) y más adelante fue corroborado por Agulló (2001), Choudhury (2002), Deshpande (1999), Mora (2011), Olmedo (2006), Ratnam (2005), Rojas y Salas (2011) y Wacquant (2008). Lo anterior se evidencia con mayor claridad en el incremento de la heterogeneidad de las relaciones laborales que se ha presentado en los mercados de trabajo de diversos países. Para Castillo:

las tendencias mundiales evidencian la expansión de diversas formas de trabajo precario, diferenciadas de las formas tradicionales de empleo a tiempo completo, con contrato definido, con empleador único y lugar fijo de 
trabajo, generando formas atípicas, "anormales" de empleo asalariado y no asalariado a todos los niveles y en distintos sectores de las ocupaciones. (2001, p. 101).

La precarización laboral es un proceso global que ha permeado en todo el mundo (Agulló 2001; Carnoy, 2001; Nachane, 2007; Shariff y Gumber, 1999; Wacquant, 2008). Según Agulló, es "un modelo laboral que ya no garantiza hoy por hoy la integración social de todos los ciudadanos". (2001, p. 91).

En este sentido, el centro de la precariedad laboral radica en el empleo precario, concepto que se introdujo paulatinamente en las discusiones sobre las relaciones de los trabajadores; uno de los precursores en estudiarlo fue Gerry Rodgers, quien vinculó al empleo precario con las formas de trabajo que caracterizaban a los trabajos inseguros e inestables.

Un aspecto relevante del deterioro de las relaciones laborales es que el empleo precario se ha extendido a sectores que antes tenían trabajos estables, con protección social y jornadas regulares (Mora y Oliveira, 2009). Según García:

desde mediados de los años 1990 consideramos que se fue haciendo cada vez más evidente que los informales o los marginales no estaban solos al enfrentar condiciones desfavorables, y que los empleos que se consideraban protegidos o formales en la industria, el comercio y los servicios también estaban sufriendo transformaciones de diversos tipos. $(2009$, p. 6).

Con base en lo anterior, se puede señalar que la precariedad laboral es un proceso asociado con el deterioro paulatino de las relaciones laborales, y que se acentuó con la reestructuración productiva y económica que dio origen a la implementación del modelo neoliberal.

En los últimos años, las relaciones laborales adversas se incrementaron, de manera que las formas de trabajo no asalariado se expandieron hacia los asalariados, lo que incidió en un número importante de quienes contaban con empleos estables y seguros; es decir, se comenzaron a manifestar en los del núcleo articulador moderno (asalariado, protegido, estable, seguro).

Así, una de las preocupaciones principales en torno al estudio del empleo precario radica en el carácter expansivo que ha adquirido, y que ha derivado en una profunda desestabilización y segmentación del trabajo.

Según Mora:

el concepto de empleo precario busca mostrar cómo la dimensión de la ciudadanía social es erosionada cuando el trabajo es conceptuado básicamente como una mercancía y el empleo es sujeto a procesos de desregulación que impiden a los trabajadores ejercer sus derechos básicos. Así, mediante el uso de este concepto se podrían observar rupturas entre procesos de crecimiento económico, reestructuración productiva e integración social. (2006, p. 33).

Uno de los planteamientos centrales de la precariedad laboral es la multidimensionalidad para acercarse a las relaciones laborales de los trabajadores. Guerra (1994); Mora (2006) y Rodgers (1989) destacan que las dimensiones de 
mayor relevancia en la precariedad laboral son la inseguridad (sin acceso a la seguridad social), la inestabilidad (falta de continuidad) y la vulnerabilidad económica (salarios bajos).

Según Agulló (2001), la precariedad es un proceso multidimensional que trasforma las relaciones laborales y se presenta en dos sentidos: a) la precariedad de la situación social, que se refiere a los trabajadores que involuntariamente tienen un empleo de tiempo parcial, son los autoempleados, y b) la precariedad de subsistencia, que corresponde a los empleados que se encuentran por debajo de los estándares mínimos de protección social. Es importante señalar que ambas facetas no son excluyentes entre sí, sino que se suelen manifestar de manera conjunta.

Mora (2006) define la noción de precariedad laboral a través de los elementos siguientes:

- Las relaciones laborales regidas por criterios de incertidumbre, donde predominan la inseguridad y la inestabilidad laboral.

- La adopción de políticas de remuneración de la fuerza laboral, regidas por criterios de minimización de costos.

- El desarrollo de estrategias de contratación que cumplen parcialmente o evaden los sistemas de seguridad social y derechos laborales.

- La definición unilateral del tiempo de trabajo en afinidad con los requerimientos productivos del sector empresarial.

Para Guerra (1994), la precariedad laboral se aborda a través de tres dimensiones: la inseguridad, la inestabilidad y los salarios bajos; con ellas intenta mostrar el debilitamiento gradual de los derechos elementales de los trabajadores asalariados.

En esta investigación, la precariedad laboral se define como resultado de una serie de cambios en la estructura social y económica, generados a escala mundial, con efectos en el mercado de trabajo, donde el abaratamiento de la mano de obra se refleja en relaciones laborales adversas para los trabajadores que se evidencian en inseguridad, inestabilidad y salarios bajos, entre otros aspectos.

Aquí, el acercamiento empírico a la precariedad laboral se realizó mediante la inseguridad, la inestabilidad y la vulnerabilidad económicas, que permiten estimarla a través de indicadores como el ingreso salarial, el acceso a la seguridad social, el tipo de contrato y el tamaño de la empresa. Es importante señalar que la revisión de la bibliografía muestra evidencia suficiente para señalar que los indicadores elegidos se vinculan con el empleo precario.

La primera relación laboral ${ }^{6}$ se eligió porque plantea que los ingresos salariales se asocian con la insuficiencia, cuando las percepciones son inferiores a las requeridas para satisfacer las necesidades básicas en alimentación, salud y vivienda, que permiten garantizarles a los trabajadores las condiciones materiales para sobrevivir. La segunda se refiere al acceso a la seguridad social, vinculada con la atención médica que garantiza la salud de los trabajadores y

6 Aquí, una relación laboral se define como las condiciones de compraventa de la fuerza de trabajo. 
su familias (Rodgers, 1989). La tercera se relaciona con el tipo de contrato de trabajo que hace referencia a la certeza del empleo (Guerra, 1994). Por último, el tamaño de la empresa se considera de suma relevancia, ya que en diversas investigaciones se encontró que es una característica del mercado de trabajo, que determina las condiciones laborales favorables o adversas (Cervantes, 2017; Guadarrama, Hualde y López-Estrada, 2012; Oliveira, 2009).

En términos generales, la elección de estas relaciones laborales se debe a que: a) son aspectos centrales del concepto de empleo precario, como lo plantean Guerra (1994); Mora (2005) y Rodgers (1989); b) captan elementos relevantes de las relaciones laborales y c) la fuente de información seleccionada (ENOE) cuenta con los datos de dichos indicadores, necesarios para lograr el objetivo de esta investigación.

Una de las limitaciones del concepto de empleo precario es que estudia solo a los trabajadores asalariados, debido a que aprehende los cambios ocurridos (principalmente) en el trabajo asalariado. Por tanto, en este artículo se analiza la precariedad laboral en dicho grupo.

No obstante, las relaciones laborales precarias también se presentan en los trabajadores no asalariados, es decir, en los que laboran por cuenta propia, y que en su mayoría caracterizan al sector informal de la economía, con ocupaciones de menor calidad, productividad, menos ingresos y relaciones de trabajo más adversas que los asalariados (Castillo, 2009). De ahí que la precariedad y la informalidad se comenzaron a analizar de manera conjunta. Sin embargo, dicha relación traspasa los límites de esta investigación.

\section{Contexto sociodemográfico y entorno laboral de los trabajadores del Estado de México, 2005 y 2015}

En este apartado primero se abordan las características sociodemográficas de los trabajadores del Estado de México, para conocer su distribución por sexo, los grupos de edad y el nivel educativo en 2005 y 2015. Después se hace referencia al entorno laboral de la población objetivo, constituida por los trabajadores asalariados mexiquenses, con la intención de presentar, a grandes rasgos, la configuración de ellos. Según Román (2013), las condiciones laborales de los trabajadores jóvenes del Estado de México ya eran adversas, debido a que 50\% no contaba con contrato escrito ni atención médica, además, en 2015 casi $60 \%$ de ellos eran informales.

El Estado de México es la entidad más poblada del país; según la ENOE, en 2015 había 16.8 millones de personas, de las cuales alrededor de 7 millones laboraban, como se muestra en la Tabla 1, donde también aparece la distribución porcentual por sexo, y los grupos de edad con mayor participación, entre los que sobresale el aumento en el de 50 a 59 años, que expone un envejecimiento de la población trabajadora. También se da cuenta de su nivel educativo, un elemento importante en este aspecto es el incremento de quienes cuentan con preparatoria, profesional y posgrado, en detrimento de la educación básica, que es consecuencia de la escolarización de los mexicanos en general. 
Tabla 1. Distribución porcentual de trabajadores del Estado de México según variables sociodemográficas, 2005 y 2015

\begin{tabular}{|c|c|c|}
\hline Variables sociodemográficas & 2005 & 2015 \\
\hline \multicolumn{3}{|l|}{ Sexo } \\
\hline Hombre & 64.8 & 62.9 \\
\hline Mujer & 35.2 & 37.1 \\
\hline \multicolumn{3}{|l|}{ Grupos de edad } \\
\hline 12 a 19 & 7.7 & 5.2 \\
\hline 20 a 29 & 26.8 & 23.1 \\
\hline 30 a 39 & 25.9 & 25.6 \\
\hline 40 a 49 & 21.5 & 22.6 \\
\hline 50 a 59 & 11.8 & 15.6 \\
\hline 60 a 69 & 4.7 & 5.8 \\
\hline 70 y más & 1.7 & 2.1 \\
\hline No especificado & 0.0 & 0.05 \\
\hline \multicolumn{3}{|l|}{ Nivel educativo } \\
\hline Ninguno & 4.2 & 2.3 \\
\hline Básico & 59.2 & 54.9 \\
\hline Preparatoria o bachillerato & 14.7 & 20.3 \\
\hline Normal o carrera técnica & 8.0 & 5.5 \\
\hline Profesional y posgrado & 13.1 & 17.0 \\
\hline No sabe & 0.1 & 0.1 \\
\hline Total & 5461979 & 7030943 \\
\hline
\end{tabular}

Fuente: elaboración propia, con datos de la ENOE (Lucatero, 2016).

Como preámbulo al análisis de la precariedad laboral, en la Tabla 2 se muestran diversos indicadores que permiten conocer la estructura del mercado laboral mexiquense y sus características. En 2005, había 10.7 millones de personas mayores de 12 años, ${ }^{7}$ para 2015 aumentaron a 13.2 millones, lo que generó un incremento de 2.5 millones de personas que se podrían incorporar al mercado de trabajo. De este grupo poblacional, la participación de los hombres en 2005 fue de 70\% de la PEA, y la de las mujeres de 35. Para 2015, la PEA de los hombres se mantuvo, sin embargo la participación femenina creció en casi $5 \%$.

Es importante mencionar que se consideraron los trabajadores a partir de los 12 años, debido a que la fuente de información genera los datos del mercado laboral a partir de esa edad. 
Tabla 2. Indicadores laborales seleccionados del Estado de México por sexo, 2005 y 2015

\begin{tabular}{|c|r|r|r|r|}
\hline \multirow{2}{*}{$\begin{array}{c}\text { Indicadores } \\
\text { seleccionados }\end{array}$} & \multicolumn{2}{|c|}{2005} & \multicolumn{2}{c|}{2015} \\
\cline { 2 - 5 } & Mujeres & Hombres & \multicolumn{1}{c|}{ Mujeres } & Mujeres \\
\hline PEA & 35.1 & 73.3 & 40.0 & 72.9 \\
\hline Ocupados & 94.8 & 94.5 & 93.5 & 95.0 \\
\hline Asalariados & 67.8 & 70.9 & 67.6 & 73.3 \\
\hline No asalariados & 32.2 & 29.1 & 32.4 & 23.7 \\
\hline Desocupados & 5.2 & 5.5 & 6.5 & 5.0 \\
\hline *PNEA & 64.9 & 26.7 & 60.0 & 27.1 \\
\hline \multirow{2}{*}{$\begin{array}{c}\text { Población de 12 } \\
\text { años y más }\end{array}$} & 5628339 & 5113541 & 6973223 & 6394088 \\
\hline
\end{tabular}

*Población no económicamente activa.

Fuente: elaboración propia, con base en la ENOE (Lucatero, 2016).

De la PEA del Estado de México en los dos años de estudio, 95\% estaba ocupada, esto plantea que en 2005 y 2015 las personas desocupadas alcanzaban 5\%; en ambos años sobresale el mayor desempleo en las mujeres. Prácticamente en toda América Latina las mujeres suelen presentar mayor desempleo que los hombres (Comisión Económica para América Latina y el Caribe, 2017). Es importante señalar que el Estado de México es de las entidades con mayor porcentaje de desempleados en el país. En los años de estudio, de las personas mexiquenses ocupadas, alrededor de $70 \%$ eran asalariadas.

\section{Metodología}

Para lograr el objetivo de la investigación, la metodología utilizada primero planteó realizar un análisis de conglomerados (AC), que consiste en agrupar datos similares para crear conjuntos, los cuales deben ser lo más heterogéneo posible (Dunteman, 1989), lo que permitió formar cuatro conjuntos de trabajadores asalariados mexiquenses, de acuerdo con sus relaciones laborales: precario alto, precario medio, precario bajo y no precario. En segundo lugar se conformaron grupos ocupacionales, para conocer la distribución de la precariedad dentro de éstos. Los cuatro niveles de precariedad se eligieron porque muestran la heterogeneidad de éstos en los trabajadores asalariados mexiquenses.

El AC es una técnica estadística multivariada que permite conjuntar las observaciones con base en la similitud que exista entre ellas, y su objetivo principal es agrupar a la población con características similares (mínima varianza), 
y que entre los conjuntos de observaciones conformadas sean lo más diversas posible (máxima varianza) (Dunteman, 1989). Los grupos que conforma el AC se pueden integrar a través de variables u observaciones, aquí los conglomerados se formaron con variables, debido a que se vinculan con la precariedad laboral. En el AC existen dos técnicas (jerárquica y no jerárquica) para agrupar a la población, con base en diversas variables.

Aquí se utilizó el método no jerárquico porque permite elegir previamente el número de conglomerados o grupos que se va a analizar, los conjuntos se forman a partir de unos centros iniciales que configuran a las observaciones, con base en la proximidad del centro inicial. Además, dicho método se recomienda para bases de datos con observaciones numerosas, como la que se utiliza en esta investigación.

Para la conformación de los cuatro niveles de precariedad en los trabajadores asalariados mexiquenses se emplearon las variables ingreso salarial, acceso a la seguridad social, tipo de contrato y tamaño de la empresa, que se vinculan con el concepto de precariedad laboral ya definido. Con estas variables, el AC conformó los niveles de precariedad, mediante la agrupación de la población con relaciones laborales similares.

En términos generales, el AC agrupa los cuatro niveles de precariedad que se conformaron con base en las variables que definen empíricamente al concepto de precariedad laboral: a) el grupo de alta precariedad, integrado por los trabajadores con el mayor número de relaciones laborales adversas; b) el de precariedad media, que tiene dos o tres de estas relaciones; c) el grupo de precarios bajos reúne a quienes tienen una o dos de ellas y d) los trabajadores no precarios, que no tienen relaciones laborales adversas.

Una vez que se obtuvieron los cuatro grupos, se analizó su distribución por conjuntos ocupacionales, con la finalidad de conocer el efecto de la precariedad laboral en las ocupaciones del mercado laboral del Estado de México en los dos años de estudio.

Aquí se clasificaron los grupos ocupacionales con base en Standing (1999), debido a que él vincula su propuesta con la inseguridad y la incertidumbre laboral como elementos esenciales dentro de los mercados de trabajo. Además, Standing clasifica a los grupos ocupacionales en: salariat (no manual calificado), proficians (no manual no calificado), coreworkers (manual calificado) y flexiworkers (manual no calificado); en esta investigación se decidió vincularlos con trabajadores manuales y no manuales, para comprenderlos mejor.

Es importante señalar que la clasificación de estos trabajadores que, en este caso, se basó en el Sistema Nacional de Ocupaciones del INEGI (2011), está relacionada con el tipo de ocupación, por ejemplo los no manuales calificados se vinculan con directivos, profesionistas, funcionarios y altas autoridades y gerentes; los no manuales no calificados, se asocian con las ocupaciones de auxiliares, administrativos, agentes de ventas y comerciantes. Para los manuales calificados se consideró a obreros, operadores de maquinarias, choferes, conductores de trasporte, y en el grupo de manuales no calificados a los trabajadores agrícolas, de servicios personales y alimentación y vigilancia. 


\section{Fuente de información}

En esta investigación se utilizaron los datos de la ENOE, obtenidos por el INEGI de manera trimestral desde 2005, con el objetivo de captar las características ocupacionales de la población a escala nacional, así como otras variables demográficas y económicas para profundizar en el análisis de los aspectos laborales.

La ENOE aplica un muestreo probabilístico, bietápico, estratificado y por conglomerados, que se divide en cinco paneles independientes, los cuales permanecen en la muestra durante cinco trimestres, y eso permite hacer estudios longitudinales a un panel de viviendas.

\section{La precariedad laboral. Un acercamiento a las relaciones laborales de los trabajadores asalariados del Estado de México, 2005 y 2015}

En esta sección se analizan, por sexo, las relaciones laborales en los trabajadores asalariados mexiquenses, en 2005 y 2015, que son: ingreso salarial, acceso a la seguridad social, tipo de contrato y tamaño de la empresa. Con base en estas variables se aplicó el análisis por conglomerados, que permitió agrupar a dichos trabajadores en cuatro niveles de precariedad, con base en las relaciones laborales: alto, medio, bajo y no precario.

\section{Ingreso salarial ${ }^{8}$}

El ingreso por trabajo es un aspecto central para las familias, debido a que esta percepción económica es el sustento principal de los hogares en México (Montoya, 2017). En este contexto, en la Tabla 3 se muestra que en 2005 en el Estado de México aproximadamente $70 \%$ de los trabajadores asalariados recibían menos de tres salarios mínimos (\$4200); en contraste, 8.5 recibía más de cinco (\$7020). Para 2015 se mantuvieron los porcentajes de las personas que recibían menos de tres salarios mínimos (\$6 390) y hubo una reducción de $5 \%$ de quienes percibían ingresos altos (\$10 515). Esta disminución en los años de estudio expone una precarización de los salarios, y refleja una caída salarial importante de los trabajadores mexiquenses (ver Tabla 3). En las últimas décadas la contención salarial ha sido general en México (Camberos y Bracamontes, 2015).

En lo que respecta a la distribución de los ingresos salariales para hombres y mujeres mexiquenses asalariados, se exponen similitudes entre los dos años. Sin embargo, es mayor la concentración de mujeres que perciben menos de un salario mínimo, esto muestra las desigualdades históricas que sufren las trabajadoras durante su vida laboral.

Aquí se considera el ingreso laboral aproximado por el salario mínimo, debido a que la ENOE tiene deficiencias serias en la captación de él. 
Tabla 3. Distribución porcentual de los trabajadores asalariados del Estado de México por nivel de ingreso y sexo, 2005 y 2015

\begin{tabular}{|l|r|r|r|r|r|r|}
\hline \multirow{2}{*}{$\begin{array}{l}\text { Nivel de ingreso } \\
\text { (en salarios mínimos) }\end{array}$} & \multicolumn{3}{|c|}{2005} & \multicolumn{3}{|c|}{2015} \\
\cline { 2 - 7 } & Hombres & Mujeres & Total & Hombres & Mujeres & \multicolumn{1}{c|}{ Total } \\
\hline Hasta 1 & 7.3 & 13.8 & 9.5 & 7.1 & 14.5 & 9.7 \\
\hline Más de 1 y hasta 2 & 25.9 & 35.3 & 29.1 & 30.2 & 34.0 & 31.6 \\
\hline Más de 2 y hasta 3 & 32.2 & 21.6 & 28.6 & 30.1 & 21.7 & 27.1 \\
\hline Más de 3 y hasta 5 & 20.8 & 16.9 & 19.5 & 15.5 & 13.3 & 14.7 \\
\hline Más de 5 & 8.8 & 7.8 & 8.5 & 3.8 & 2.5 & 3.4 \\
\hline No especificado & 5.0 & 4.6 & 4.8 & 13.4 & 14.0 & 13.6 \\
\hline Total & 2510217 & 1302457 & 3812674 & 3243486 & 17623335005819 \\
\hline
\end{tabular}

Fuente: elaboración propia, con datos de la ENOE (Lucatero, 2016).

\section{Acceso a la seguridad social}

En 2005, 50\% de la población asalariada mexiquense contaba con acceso a alguna institución de salud por parte de su trabajo. En 2015 se mantuvo este porcentaje, lo que implica que en 10 años los avances fueron nulos en la cobertura de salud (ver Tabla 4). Los hombres contaban con mayor acceso a la seguridad social que las mujeres, esto sugiere que las condiciones laborales de ellas son más adversas.

Tabla 4. Distribución porcentual de los trabajadores asalariados del Estado de México por acceso a la seguridad social y sexo,

2005 y 2015

\begin{tabular}{|l|c|c|c|c|c|c|}
\hline \multirow{2}{*}{$\begin{array}{l}\text { Instituciones de } \\
\text { atención médica }\end{array}$} & \multicolumn{3}{|c|}{2005} & \multicolumn{3}{c|}{2015} \\
\cline { 2 - 7 } & Hombres & Mujeres & Total & Hombres & Mujeres & Total \\
\hline${ }^{*}$ IMSS & 38.7 & 36.1 & 37.9 & 40.9 & 38.2 & 40.0 \\
\hline${ }^{*}$ ISSSTE & 10.6 & 15.6 & 12.3 & 9.8 & 14.3 & 11.4 \\
\hline Otras & 1.8 & 1.8 & 1.8 & 0.4 & 0.3 & 0.4 \\
\hline $\begin{array}{l}\text { No recibe } \\
\text { atención médica }\end{array}$ & 48.6 & 46.4 & 47.8 & 48.7 & 46.8 & 48.1 \\
\hline No especificado & 0.3 & 0.1 & 0.2 & 0.1 & 0.3 & 0.2 \\
\hline Total & 2510217 & 1302457 & 3812674 & 3243486 & 1762333 & 5005819 \\
\hline
\end{tabular}

*Instituto Mexicano del Seguro Social.

**Instituto de Seguridad y Servicios Sociales de los Trabajadores del Estado

Fuente: elaboración propia, con datos de la ENOE (Lucatero, 2016). 


\section{Tipo de contrato laboral}

En los dos años de estudio, aproximadamente $50 \%$ de la población mexiquense asalariada carecía de contrato (ver Tabla 5). A través del tiempo, solo se ha reducido $3 \%$ la proporción de trabajadores sin contrato, lo que señala que en 10 años los avances en este derecho fueron limitados. De acuerdo con García (2010), las personas que laboran sin contrato constituyen un signo de la precariedad progresiva del trabajo en una región.

Tabla 5. Distribución porcentual de los trabajadores asalariados del Estado de México por tipo de contrato y sexo, 2005 y 2015

\begin{tabular}{|l|c|c|c|c|c|c|}
\hline \multirow{2}{*}{$\begin{array}{l}\text { Tipo } \\
\text { de contrato }\end{array}$} & \multicolumn{3}{|c|}{2005} & \multicolumn{3}{c|}{2015} \\
\cline { 2 - 7 } & Hombres & Mujeres & Total & Hombres & Mujeres & Total \\
\hline Temporal & 9.2 & 11.9 & 10.1 & 10.6 & 11.0 & 10.7 \\
\hline $\begin{array}{l}\text { De base, planta } \\
\text { o por tiempo } \\
\text { indefinido }\end{array}$ & 40.5 & 41.6 & 40.8 & 42.4 & 44.2 & 43.0 \\
\hline No especificado & 0.2 & 0.2 & 0.2 & 0.3 & 0.2 & 0.2 \\
\hline $\begin{array}{l}\text { Sin contrato } \\
\text { escrito }\end{array}$ & 49.4 & 45.8 & 48.2 & 46.4 & 44.3 & 45.7 \\
\hline No especificado & 0.7 & 0.6 & 0.6 & 0.3 & 0.3 & 0.3 \\
\hline Total & 2510217 & 1302457 & 3812674 & 3243486 & 1762333 & 5005819 \\
\hline
\end{tabular}

Fuente: elaboración propia, con datos de la ENOE (Lucatero, 2016).

Los hombres tienen mayor incertidumbre laboral, porque una parte importante de las mujeres se incorpora al mercado laboral en ocupaciones asociadas con la salud y la educación, sectores caracterizados por generar empleos con buenas condiciones laborales.

\section{Tamaño de la empresa}

En la mayoría de los casos, las empresas con más empleados están asociadas con más derechos y prestaciones, lo que se traduce en mayor estabilidad laboral (García, 2013).

En los dos años de estudio, el porcentaje de trabajadores asalariados de microempresas (menos de seis) alcanzó alrededor de 34\% (ver Tabla 6). Un aspecto relevante de estos resultados es el alto porcentaje de mujeres en empresas con un solo trabajador, lo que implica que las mexiquenses son las más perjudicadas en diferentes frentes de las relaciones laborales, lo que expone un proceso de precarización hacia ellas, que se acentúa a través de salarios bajos. 
Tabla 6. Distribución porcentual de los trabajadores asalariados del Estado de México por tamaño de la empresa y sexo, 2005 y 2015

\begin{tabular}{|l|c|c|c|c|c|c|}
\hline \multirow{2}{*}{$\begin{array}{l}\text { Tamaño de la } \\
\text { empresa } \\
\text { (número } \\
\text { de personas) }\end{array}$} & \multicolumn{3}{|c|}{2005} & \multicolumn{3}{c|}{2015} \\
\cline { 2 - 7 } & Hombres & Mujeres & Total & Hombres & Mujeres & Total \\
\hline 1 & 0.1 & 12.0 & 4.1 & 0.2 & 12.1 & 4.3 \\
\hline De 2 a 5 & 33.5 & 21.3 & 29.4 & 33.6 & 22.5 & 29.6 \\
\hline De 6 a 10 & 9.2 & 9.4 & 9.2 & 9.2 & 8.2 & 8.9 \\
\hline De 11 a 15 & 3.9 & 5.4 & 4.5 & 4.1 & 4.8 & 4.4 \\
\hline De 16 a 50 & 15.6 & 19.0 & 16.8 & 18.0 & 20.0 & 18.7 \\
\hline De 51 y más & 34.7 & 31.0 & 33.5 & 33.5 & 31.9 & 33.0 \\
\hline No especificado & 2.9 & 1.9 & 2.6 & 1.5 & 0.6 & 1.1 \\
\hline Total & 2510217 & 1302457 & 3812674 & 3243486 & 1762333 & 5005819 \\
\hline
\end{tabular}

Fuente: elaboración propia, con datos de la ENOE (Lucatero, 2016).

El análisis anterior tiene limitaciones, pues solo se conocen de forma aislada las relaciones laborales que afectan a la población asalariada mexiquense en los años de estudio, por lo que a continuación se expone el resultado del $A C$ en el que se muestran los cuatro niveles (precario alto, precario medio, precario bajo y no precario), con estos grupos se pretende conocer la precariedad de los trabajadores asalariados del Estado de México en 2005 y 2015.

\section{Niveles de precariedad laboral y grupos ocupacionales en el Estado de México, 2005 y 2015}

En 2005, aproximadamente dos tercios de los trabajadores asalariados del Estado de México tenía precariedad laboral; casi 35\% de ellos alcanzaban precariedad alta, lo que indica que una tercera parte padecía inseguridad económica e inestabilidad laboral, lo que repercute en los derechos laborales básicos, así como en sus condiciones materiales (ver Tabla 7). En contraste, en este mismo año cerca de $30 \%$ de los asalariados no tenía relaciones laborales precarias, más bien eran favorables. Esto significa una gran presencia de trabajadores con diferencias relevantes (precario alto y no precario), lo que se podría traducir en una polarización, que acentúa la desigualdad laboral. 
Tabla 7. Distribución porcentual de trabajadores asalariados del Estado de México según nivel de precariedad, 2005

\begin{tabular}{|c|r|}
\hline Precario alto & 34.8 \\
\hline Precario medio & 13.0 \\
\hline Precario bajo & 23.2 \\
\hline No precario & 28.9 \\
\hline Total & 3529766 \\
\hline
\end{tabular}

Fuente: cálculos propios, con base en la ENOE (Lucatero, 2016).

En 2015, 65\% de la población mexiquense asalariada tenía algún nivel de precariedad (ver Tabla 8), lo que implica un mercado laboral altamente precarizado; los no precarios alcanzaban $35 \%$. En este año también se expone una división (precario/no precario), que plantea una desigualdad persistente en la población asalariada, los más afectados fueron quienes tenían un empleo con precariedad alta.

Tabla 8. Distribución porcentual de trabajadores asalariados del Estado de México según nivel de precariedad, 2015

\begin{tabular}{|c|r|}
\hline Precario alto & 36.7 \\
\hline Precario medio & 12.3 \\
\hline Precario bajo & 16.0 \\
\hline No precario & 35.0 \\
\hline Total & 4271530 \\
\hline
\end{tabular}

Fuente: cálculos propios, con base en la ENOE (Lucatero, 2016).

Un elemento común que sobresale en el análisis de los resultados de la precariedad laboral en 2005 y 2015 es un mercado de trabajo mexiquense fragmentado entre trabajadores precarios y no precarios, que apunta a una polarización (Mora y Oliveira, 2009; Román, 2013).

En dicho contexto, esta investigación plantea profundizar en el estudio de la precariedad laboral mediante el efecto que causa en los grupos ocupacionales, para conocer a los más perjudicados. Las ocupaciones se conformaron en cuatro grupos: no manual calificado, no manual no calificado, manual calificado y manual no calificado (ver Tabla 9).

Los resultados indican que $40 \%$ de los trabajadores asalariados precarios altos se concentraba en el grupo de manual no calificado. Este porcentaje se esperaba debido a que este conjunto se asocia tradicionalmente con relaciones laborales adversas. En contraste, en 2005 más de la mitad de los asalariados mexiquenses no precarios se concentraba en el grupo no manual calificado, esto plantea que 
la precariedad laboral ha golpeado a una parte de los empleos con mejores niveles educativos, lo que coincide con Guerra (1994) sobre una expansión de este fenómeno en la estructura del mercado de trabajo.

En lo que respecta a los niveles de precario medio y precario bajo, en 2005 predominaban los grupos intermedios (no manual no calificado y manual calificado), esto plantea una distribución heterogénea de los trabajadores por niveles de precariedad (ver Tabla 9).

Tabla 9. Distribución porcentual de trabajadores asalariados por nivel de precariedad y grupo ocupacional, Estado de México, 2005

\begin{tabular}{|c|r|r|r|r|}
\hline $\begin{array}{c}\text { Nivel de } \\
\text { precariedad }\end{array}$ & $\begin{array}{c}\text { No manual } \\
\text { calificado }\end{array}$ & $\begin{array}{c}\text { No manual } \\
\text { no calificado }\end{array}$ & $\begin{array}{c}\text { Manual } \\
\text { calificado }\end{array}$ & $\begin{array}{c}\text { Manual } \\
\text { no calificado }\end{array}$ \\
\hline Precario alto & 42.2 & 55.9 & 6.0 & 29.4 \\
\hline Precario medio & 8.8 & 17.5 & 7.5 & 16.7 \\
\hline Precario bajo & 15.3 & 6.1 & 63.4 & 17.8 \\
\hline No precario & 33.7 & 20.5 & 23.1 & 36.1 \\
\hline Total & 922185 & 897946 & 700022 & 1009613 \\
\hline
\end{tabular}

Fuente: elaboración propia, con datos de la ENOE (Lucatero, 2016).

Para 2015 , casi $65 \%$ de los asalariados mexiquenses con precariedad alta se encontraban en el grupo de manual no calificado (ver Tabla 10), que concentraba a gran parte de los trabajadores con algún nivel de precariedad, lo que implica que el fenómeno está fuertemente inserto en él.

Tabla 10. Distribución porcentual de trabajadores asalariados por nivel de precariedad y grupo ocupacional, Estado de México, 2015

\begin{tabular}{|c|r|r|r|r|}
\hline $\begin{array}{c}\text { Nivel de } \\
\text { precariedad }\end{array}$ & $\begin{array}{c}\text { No manual } \\
\text { calificado }\end{array}$ & $\begin{array}{c}\text { No manual } \\
\text { no calificado }\end{array}$ & $\begin{array}{c}\text { Manual } \\
\text { calificado }\end{array}$ & $\begin{array}{c}\text { Manual } \\
\text { no calificado }\end{array}$ \\
\hline Precario alto & 33.1 & 55.9 & 7.4 & 27.8 \\
\hline Precario medio & 11.5 & 13.9 & 9.8 & 12.1 \\
\hline Precario bajo & 10.3 & 5.2 & 46.9 & 14.9 \\
\hline No precario & 45.1 & 25.0 & 35.9 & 45.1 \\
\hline Total & 822217 & 1782458 & 811801 & 855054 \\
\hline
\end{tabular}

Fuente: elaboración propia, con datos de la ENOE (Lucatero, 2016).

En 2015, los trabajadores asalariados del Estado de México con relaciones laborales no precarias estaban concentrados en el grupo de los no manuales calificados; sin embargo, sobresale que éste contaba con porcentajes de preca- 
riedad baja y media. Estos resultados plantean una precarización del empleo en todos los grupos, situación que de alguna manera se confirma con los trabajos anteriores sobre este tema (Rojas y Salas, 2011).

En concreto, en los resultados se identifica que la precariedad laboral perjudica mucho a los trabajadores asalariados mexiquenses, en particular al grupo de los manuales no calificados. Por tanto, dichos resultados se podrían entender y explicar dada la estructura económica que prevalece en el Estado de México. Del total de empresas registradas en la entidad, $95.8 \%$ son micro, 3.31 son pequeñas, 0.43 son medianas y 0.46 son grandes (Lucatero, 2016); con lo que se observa un mayor porcentaje de micro y pequeños establecimientos, incluso superior al nacional, que dan cabida a los trabajadores en ocupaciones asociadas con los servicios personales y alimentación (manuales no calificados), y por lo tanto relaciones laborales más adversas.

Otra de las explicaciones posibles radica en que uno de cada dos ocupados es joven (menor de 30 años) y la escolaridad de tres de cada cuatro no supera el nivel medio superior. Además, el excedente de población que reside en el Estado de México hace que la demanda laboral sea más selectiva, y que pocos trabajadores obtengan las ocupaciones no manuales calificadas, para con ello acceder a relaciones laborales mejores.

\section{Conclusiones}

De acuerdo con los resultados de esta investigación, en 2005 y 2015 en el mercado laboral mexiquense se presentaron elementos de precariedad asociados con la inseguridad, la inestabilidad y la vulnerabilidad económicas. De los aspectos de mayor relevancia para los trabajadores asalariados mexiquenses en esos años, la extensión de la precariedad a todos los grupos ocupacionales es lo que les afecta, sin importar el nivel educativo y el tipo de trabajo que tengan. En particular, se expone una mayor inserción de la precariedad en los grupos asociados con directivos, profesionistas, gerentes (no manuales calificados). Lo que presenta modalidades nuevas de precariedad que inciden en los de escolaridad alta, es decir, el fenómeno se ha insertado en ocupaciones que antes se caracterizaban por contar con relaciones laborales favorables para el trabajador.

El segundo permite sugerir que el empleo producido en el Estado de México entre los años de estudio es esencialmente precario; lo que modifica la dinámica empleado-empleador, en detrimento del primero.

El tercero sugiere que el mercado laboral mexiquense presenta una división entre el trabajador precario y no precario, que agudiza la desigualdad de la estructura laboral estatal. Esto conlleva una serie de riesgos sociales, debido a que la desigualdad laboral genera problemáticas que inciden en la calidad de vida de la sociedad.

El cuarto muestra a la polarización laboral como un elemento social en el mercado de trabajo del Estado de México, debido a que casi dos terceras partes de los trabajadores se concentran en los dos extremos de la precariedad laboral (precario alto y no precario). 
Por último, se sugiere realizar un estudio a futuro para profundizar en el análisis de la precariedad laboral como fenómeno trascendental en el mercado de trabajo mexiquense. Esta línea de investigación se asocia con el análisis de las múltiples y complejas relaciones de la precariedad, la desigualdad y la polarización en el mercado de trabajo del Estado de México, con la finalidad de esclarecer las consecuencias sociales que se podrían derivar de los vínculos de estos tres fenómenos.

\section{Referencias}

Agulló, E. (2001). Entre la precariedad laboral y la exclusión social: los otros trabajos, los otros trabajadores. Madrid: Pirámide.

Camberos, M. y Bracamontes, J. (2015). La caída de los salarios en México 19802010: la productividad y la informalidad como explicación. Equilibrio Económico, Revista de Economía, Política y Sociedad, (40), 169-194.

Carnoy, M. (2001). La transformación del trabajo en la nueva economía global. El trabajo flexible en la era de la información. Madrid: Alianza.

Castillo, D. (2001). Los nuevos precarios, ¿mujeres u hombres? Tendencias en el mercado de trabajo urbano en Panamá, 1982-1999. Papeles de Población, (27), 99-145.

Castillo, D. (2009). Los nuevos trabajadores precarios. México: Miguel Ángel Porrúa.

Cervantes, D. (2017). Inestabilidad laboral en México: análisis de trayectorias, movilidad e impacto del tipo de contratación, 2005-2015. (Tesis de doctorado inédita). Universidad Nacional Autónoma de México (UNAM), Ciudad de México.

Choudhury, B. (2002). Alternative economic survey 2001-2002: Economic reforms: Development denied. Nueva Delhi: Rainbow Publishers.

Comisión Económica para América Latina y el Caribe. (Marzo, 2017). Mujeres: las más perjudicadas por el desempleo. (Notas para la igualdad No. 22.). Observatorio de Igualdad de Género de América Latina y el Caribe. Recuperado de https://oig.cepal.org/es/notas/nota-la-igualdad-ndeg-22-mujeres-mas-perjudicadas-desempleo

Deshpande, L. (1999). Labour standards and structural adjustment. Indian Journal of Labour Economics, 42(1).

Dunteman, G. (1989). Principal components analysis. Newbury Park: Sage Publications.

Farcomeni, A., Nardi, A. y Fabrizi, E. (2011). Joint analysis of occurrence and time to stability after entrance into the Italian labour market: An approach based on a Bayesian cure model with structured stochastic search variable selection. Journal of Applied Statistics, 38(11), 2627-2646.

Frobel, F., Heinrichs, J. y Kreye, O. (1981). La nueva división internacional del trabajo: paro estructural en los países industrializados e industrialización de los países en desarrollo. México: Siglo XXI. 
García, B. (2009). Los mercados de trabajo urbanos de México a principios del siglo XXI. Revista Mexicana de Sociología, 71(1), 5-46.

García, B. (2010). Población económicamente activa: evolución y perspectivas. En B. García y M. Ordorica (coords.), Los grandes problemas de México, I. Población (pp. 363-392). México: El Colegio de México.

García, B. (2013). Precariedad laboral y desempleo en México. En L. M. Valdés (coord.), Hacia una nueva ley general de población (pp. 157-180). México: UNAM. Recuperado de https://biblio.juridicas.unam.mx/bjv/detalle-libro/3538-hacia-una-nueva-ley-general-de-poblacion

Guadarrama, R., Hualde, A. y López-Estrada, S. (2012). Precariedad laboral y heterogeneidad ocupacional: una propuesta teórico-metodológica. Revista Mexicana de Sociología, 74(2), 213-243.

Guerra, P. (1994). El empleo precario y el empleo atípico: revisión bibliográfica y propuesta para el debate. Santiago de Chile: Programa de Economía del Trabajo.

Instituto Nacional de Estadística y Geografía (INEGI). (2002). Glosario. Recuperado de http://www.inegi.org.mx/est/contenidos/espanol/sistemas/cem07/ texcom/glosario/glosario.htm

Instituto Nacional de Estadística y Geografía (INEGI). (2011). Sistema Nacional de Clasificación de Ocupaciones (SINCO). Disponible en: http://www3.inegi. org. $\mathrm{mx} /$ sistemas/clasificaciones/sinco/sinco.aspx

Kalleberg, A. (2009). Precarious work, insecure workers: Employment relations in transition. American Sociological Review, 74, 1-22.

Lucatero, A. (2016). Análisis de la precariedad laboral de los trabajadores asalariados del Estado de México, 2015. (Tesis de licenciatura). Universidad Autónoma del Estado de México.

Marx, K. (1991). El capital (17 ${ }^{a}$ ed., vol. I). México: Siglo XXI.

Montoya, V. (2017). Los hogares en la crisis: trabajo y condiciones de vida en México, 2008-2010. México: Comisión Económica para América Latina y el Caribe y UNAM.

Mora, M. (2005). Ajuste y empleo: notas sobre la precarización del empleo asalariado. Revista de Ciencias Sociales, II(108), 27-40.

Mora, M. (2006). Ajuste estructural y empleo precario: el caso de Costa Rica. (Tesis de doctorado en ciencia social inédita). El Colegio de México, Ciudad de México.

Mora, M. (2011). El empleo precario asalariado y globalización: enseñanzas desde Costa Rica. En E. Pacheco Muñoz, E. de la Garza y L. Reygadas (coords.), Trabajos atípicos y precarización del empleo (pp. 161-198). México: El Colegio de México.

Mora, M. y Oliveira de, O. (2009). La degradación del empleo asalariado en los albores del siglo XXI: Costa Rica y México. Papeles de Población, (61), 195231.

Nachane, D. (2007). Two decades of structural reforms in India: A balance sheet. Pune: Gokhale Institute of Politics and Economics.

Olmedo, C. (2006). Flexibilización laboral: informalización-precarización del empleo: el Estado en el centro del debate. Sociología del Trabajo, (58), 115136. 
Oularis, S. (2011). ¿Qué son los modelos económicos? Finanzas \& Desarrollo, (48), 46-47.

Ratnam, V. (2005). Labour market in India. En B. Debroy y P. D. Kaushik (Eds.), Reforming the labour market. Nueva Delhi: Academic Foundation.

Rodgers, G. (1989). Precarious work in Western Europe: The state of the debate. En G. Rodgers y J. Rodgers (Eds.), Precarious jobs in labour market regulation: The growth of atypical employment in Western Europe (pp. 1-16). Génova: International Institute for Labour Studies y Free University of Brussels.

Rojas, G. y Salas, C. (2011). Precariedad laboral y la estructura del empleo en México, 1995-2004. En E. Pacheco Muñoz, E. de la Garza y L. Reygadas (coords.), Trabajos atípicos y precarización del empleo (pp. 117-160). México: El Colegio de México.

Román, Y. (2013). Impactos sociodemográficos y económicos en la precariedad laboral de los jóvenes en México. región y sociedad, 25(58), 165-202. doi: 10.22198/rys.2013.58.a127

Rubio, J. (2017). Sindicalización y precariedad laboral en México. región y sociedad, 29(68), 37-75. doi: 10.22198/rys.2017.68.a247

Shariff, A. y Gumber, A. (1999). Employment and wages in India: Pre and post reform scenario. Indian Journal of Labour Economics, 42(2), 195-216.

Sollova, V. y Barrios, A. (2010). La dinámica laboral y la estructura demográfica en el Estado de México, durante el año 2010. Revista Trimestral de Análisis de Coyuntura Económica, III(4), 14-16.

Stampini, M. y Verdier A. (2011). Labor market dynamics in Tunisia: The issue of youth unemployment (The Working Paper Series No. 123). African Development Bank, Tunis, Tunisia.

Standing, G. (1999). Global labour flexibility: Seeking distributive justice. Nueva York: St. Martin's Press.

Wacquant, L. (2008). Los condenados de la ciudad: gueto, periferias y Estado. Buenos Aires: Siglo XXI. 